\title{
Cognitive Shifting in Children with Attention-Deficit Hyperactivity Disorder: A Near Infrared Spectroscopy Study
}

 \\ ${ }^{1}$ Department of Developmental Disorders, National Institute of Mental Health, National Center of Neurology and Psychiatry, Japan \\ ${ }^{2}$ Department of Children, Saitama Jyunshin College, Japan \\ ${ }^{3}$ Department of School Education, Joetsu University of Education, Japan \\ ${ }^{4}$ Graduate School of Arts and Sciences, The University of Tokyo, Japan \\ ${ }^{5}$ Department of Child Neurology, NCNP Hospital, Tokyo, Japan
}

*Corresponding author: Akira Yasumura, Department of Developmental Disorders, National Institute of Mental Health, National Center of Neurology and Psychiatry (NCNP), 4-1-1 Ogawahigashi, Kodaira, Tokyo 187-8553, Japan, Tel.: +81 42341 2711; FAX: +81 42346 2158; E-mail: yasumura@ncnp.go.jp

Received Date: September 13, 2014, Accepted Date: October 22, 2014, Published Date: October 30, 2014

\begin{abstract}
Background: Executive function is an assemblage of high-level cognitive domains (inhibition, working memory, planning, fluency, and shifting) that facilitate the inhibition of incorrect behaviors and the selection of appropriate behaviors according to context and goals. A deficit in cognitive shifting might be a core symptom of attention-deficit hyperactivity disorder. However, previous studies addressing this issue used neurobehavioral tests, which might not be specific enough to measure cognitive shifting. The purpose of the present study was to compare cognitive shifting among children with attention-deficit hyperactivity disorder and typically developing children using a Dimensional Change Card Sort task while simultaneously measuring patterns of neural activity in the prefrontal cortex.
\end{abstract}

Methods: We recruited 22 children diagnosed with attention-deficit hyperactivity disorder and 37 typically developing children as controls matched on age, gender, language ability, and non-verbal intelligence quotient. Participants performed the computer-based Dimensional Change Card Sort task, while prefrontal cortical activity was measured with near-infrared spectroscopy.

Results: Children with attention-deficit hyperactivity disorder exhibited more incorrect answers, similar reaction times, and less prefrontal activity as compared to the typically developing children.

Conclusion: The Dimensional Change Card Sort task results revealed deficits in cognitive shifting among children with attention-deficit hyperactivity disorder. This was particularly observed through the appearance of several performance errors and with decreases in prefrontal brain activity.

Keywords: Attention-deficit hyperactivity disorder; Executive function; Cognitive shifting; Frontal lobe function; Near-infrared spectroscopy (NIRS); Children

\section{Introduction}

Attention-deficit hyperactivity disorder (ADHD) is a developmental disease characterized by inattention, hyperactivity, and impulsivity [1]. Several studies have shown that core ADHD symptoms are related to deficits in executive function [2,3]. Executive function is an assemblage of high-level cognitive domains, including inhibition, working memory, planning, fluency, and shifting, that facilitate the inhibition of incorrect behaviors and the selection of appropriate behaviors according to context and goals [4]. Cognitive shifting is the mental ability to switch between thinking about one concept and begin thinking of another; it is often measured using the Wisconsin Card Sorting Test [5-8]. Kado et al. [6] previously demonstrated that children with ADHD make many more Nelsontype preservative errors (PENs) than do typically developing children (TDC). PENs are persistently repeated responses in accordance with a patient's initial preference or card sorting pattern that would have been correct during the immediately preceding stage or during the first stage of the task. During the WCST, participants need to change their responses according to shape, color, or number following feedback from the experimenter. However, using the WCST as a specific measure of cognitive shifting is problematic because this task requires additional cognitive processes, thus making it hard to elicit a specific shifting effect. Conversely, the Dimensional Change Card Sort (DCCS) task is specific to cognitive shifting and entails instructions that are easily understood by individuals from a wide range of ages and intellectual abilities [9]. In a previous study using DCCS, 3-year-old children tended to respond to a new stimulus in the same manner as an old stimulus, despite changes in rules or goals. This lack of behavioral flexibility has also been observed among older children and adults with damage to the prefrontal cortex. In contrast, typically developing 5-year-old children alter their responses according to rule changes [10]. Therefore, the DCCS task can measure developmental changes in executive function. A recent study also demonstrated that children with ADHD experienced difficulty in a modified DCCS task with more frequent rule switches [11].

In addition to deficits in the modified DCCS task, some ADHD patients display weaker prefrontal cortex (PFC) activation during a stop-signal task [12]. Recent neuroanatomical research suggests that 8to 12 -year-old children with ADHD exhibit a marked delay in the 
Citation: Yasumura A, Yamamoto H, Yasumura Y, Moriguchi Y, Hiraki K, et al. (2015) Cognitive Shifting in Children with Attention-Deficit Hyperactivity Disorder: A Near Infrared Spectroscopy Study. J Psychiatry 18: 196. doi:10.4172/2378-5756.1000196

Page 2 of 8

maturation of prefrontal areas [13]. These studies suggest that patients with ADHD may have functional and anatomical deficits in the prefrontal cortex. However, there are few brain-imaging studies of young children with ADHD. Simultaneous neurobehavioral testing using DCCS and neuroimaging may help determine why older children with ADHD exhibit the same deficits in cognitive shifting observed in very young TDC. In the present study, we evaluated cognitive shifting in children with ADHD during the DCCS task while simultaneously measuring patterns of PFC activity using near-infrared spectroscopy (NIRS)-a non-invasive measure of cerebral blood oxygenation that does not require children to be in a fixed position. Therefore, this method can be used to assess young, hyperactive children $[15,16]$. We hypothesized that children with ADHD would make more errors during a cognitive shifting task than would TDC due to the impulsivity associated with ADHD. However, we posited that children with ADHD would demonstrate similar reaction times on the task as compared to TDC.

\section{Method}

\section{Participants}

The ADHD group (Table 1) consisted of 22 participants (mean age: 10 years 3 months, standard deviation: 2 years 0 months, 15 boys and 7 girls, all but three right-handed) who were diagnosed by 2 pediatric neurologists according to DSM-IV-TR criteria [1]. The IQs of the ADHD group were evaluated using the third edition of the Japanese version of the Wechsler Intelligence Scale for Children [17]. No individual had an IQ score lower than 80. A total of 37 TDC (mean age: 10 years 10 months, standard deviation: 1 years 8 months, 19 boys and 18 girls, all but four right-handed) were recruited as controls. All participants had normal or corrected-to-normal vision and no history of neurological disorders. All participants from both groups and their mothers provided written informed consent before the experiment, the protocol for which was approved by the Ethics Committee of the National Center of Neurology and Psychiatry (Japan). We gathered the clinical sample from the National Center of Neurology and Psychiatry (Japan) and the control sample from an after-school care program at Sukage Nursery.

All participants completed Raven's Colored Progressive Matrices (RCPM) [18] to determine non-verbal intelligence. In addition, we measured sentence comprehension using the Kaufman Assessment Battery for Children (K-ABC). The Swanson, Nolan, and Pelham Scale version IV (SNAP-IV) [19-21] was completed by the mother of each participant in the TDC and ADHD groups in order to verify both the current severity of ADHD symptoms and the absence of symptoms in the control group. The SNAP-IV test assessed three cognitive/ behavioral deficits: the inattention domain, the hyperactivity/ impulsivity domain, and the oppositional defiant disorder (ODD) domain. The Pervasive Developmental Disorders Autism Society Japan Rating Scale [22] was also administered by interviewing the mother of each participant in the TDC and ADHD groups to verify ASD severity since ADHD and ASD are often combined. The PARS test included two question categories: questions about clinical conditions during infancy (PARS-Infant) and questions about current clinical conditions (PARS-Present). There were no significant differences between groups in mean age, $\mathrm{K}-\mathrm{ABC}$ sentence comprehension score, or RCPM nonverbal intelligence score (age: $\mathrm{t}(57)=1.13$, n.s.; reading comprehension: $\mathrm{t}(57)=1.61$, n.s.; RCPM: $\mathrm{t}(57)=0.96$, n.s.; see Table 1). However, significant differences were observed between groups on the three SNAP-IV subscores (inattention: $\mathrm{t}(57)=7.01, \quad \mathrm{p}<0.001 ; \quad$ hyperactivity/impulsivity: $\mathrm{t}(57)=5.76, \mathrm{p}<0.001$; ODD: $\mathrm{t}(57)=3.69, \mathrm{p}<0.01$; Table 1). Moreover, significant differences were observed between groups on the two PARS subscores (Infant: $\mathrm{t}(57)=6.72, \mathrm{p}<0.001$; Present: $\mathrm{t}(57)=7.20, \mathrm{p}<0.001$; Table 1).

n.s. $=$ non-significant: $\mathrm{p}>0.05$

\begin{tabular}{|c|c|c|c|c|}
\hline & $\begin{array}{l}\text { TDC } \\
(n=37)\end{array}$ & $\begin{array}{l}\text { ADHD } \\
(n=22)\end{array}$ & $\begin{array}{l}t \text { value } \\
\text { (df }=57 \text { ) }\end{array}$ & $p$ value \\
\hline Age & $\begin{array}{l}10: 10 \\
(1: 8)\end{array}$ & $\begin{array}{l}10: 3 \\
(2: 0)\end{array}$ & 1.13 & .263 \\
\hline $\begin{array}{l}\text { Reading } \\
\text { comprehension } \\
\mathrm{ABC} \text { a } \mathrm{K} \text { - }\end{array}$ & $\begin{array}{l}19.84 \\
(3.81)\end{array}$ & $\begin{array}{l}17.91 \\
(5.38)\end{array}$ & 1.61 & .113 \\
\hline $\begin{array}{l}\text { Non-verbal } \\
\text { intelligence } \\
\text { (RCPM) b }\end{array}$ & $\begin{array}{l}29.54 \\
(3.86)\end{array}$ & $\begin{array}{l}30.50 \\
(3.50)\end{array}$ & 0.96 & .343 \\
\hline $\begin{array}{l}\text { Inattention } \\
\text { (SNAP) c }\end{array}$ & $\begin{array}{l}5.81 \\
(4.36)\end{array}$ & $\begin{array}{l}15.73^{\star \star *} \\
(6.51)\end{array}$ & 7.01 & $<.001$ \\
\hline $\begin{array}{l}\text { Hyperactivity/ } \\
\text { impulsivity } \\
\text { (SNAP) d }\end{array}$ & $\begin{array}{l}2.05 \\
(2.38)\end{array}$ & $\begin{array}{l}8.64^{* \star *} \\
(6.26)\end{array}$ & 5.76 & $<.001$ \\
\hline $\begin{array}{l}\text { ODD } \\
\text { (SNAP) e }\end{array}$ & $\begin{array}{l}3.16 \\
(3.74)\end{array}$ & $\begin{array}{l}8.36^{* *} \\
(7.11)\end{array}$ & 3.69 & $<.01$ \\
\hline $\begin{array}{l}\text { PARS } \\
\text { (infant) } f\end{array}$ & $\begin{array}{l}1.32 \\
(1.68)\end{array}$ & $\begin{array}{l}7.18^{\star \star \star} \\
(4.86)\end{array}$ & 6.72 & $<.001$ \\
\hline $\begin{array}{l}\text { PARS } \\
\text { (present) g }\end{array}$ & $\begin{array}{l}1.51 \\
(2.22)\end{array}$ & $\begin{array}{l}8.36^{* * *} \\
(5.05)\end{array}$ & 7.20 & $<.001$ \\
\hline
\end{tabular}

Table 1: Characteristics of typically developing children (TDC) and children with attention-deficit hyperactivity disorder (ADHD).

\section{Differences were assessed using Student's t-tests (two-tailed)}

${ }^{\text {a }}$ raw score on reading comprehension as measured by the Kaufman Assessment Battery for Children (K-ABC)

braw score on Raven's Colored Progressive Matrices test (RCPM) for non-verbal intelligence

craw score on the Swanson, Nolan, and Pelham Scale (SNAP-IV) questionnaire items on inattention

${ }^{d}$ raw score on SNAP-IV questionnaire items on hyperactivity/ impulsivity

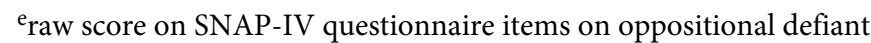
disorder

${ }^{f}$ raw score on infant items of the Pervasive Developmental Disorders Autism Society Japan Rating Scale(PARS)

graw score on present items of the PARS 
Citation: Yasumura A, Yamamoto H, Yasumura Y, Moriguchi Y, Hiraki K, et al. (2015) Cognitive Shifting in Children with Attention-Deficit Hyperactivity Disorder: A Near Infrared Spectroscopy Study. J Psychiatry 18: 196. doi:10.4172/2378-5756.1000196

\section{Behavioral task}

Each participant performed the DCCS task on a computer. Participants sat $50 \mathrm{~cm}$ away from a 15-inch liquid crystal display (LCD) screen with a gray background and performed a series of baseline trials and DCCS task trials.

\section{Baseline task}

A white square was shown on the lower right or lower left side of the 15 -inch LCD screen, and participants indicated which side the square appeared by pressing the corresponding arrow button (Figure 1A). The next square appeared randomly $1 \mathrm{~s}$ after the participants pressed the button. A 15-s rest period was allowed before beginning the task; during this time, a small white circle was shown in the center of the screen, and participants were instructed to watch it closely. Task duration was $30 \mathrm{~s}$.

\section{DCCS task}

We used the "border version" of the DCCS task to evaluate cognitive shifting among participants. The task consists of participants having to frequently alternate their responses according to the color or shape of the stimulus in line with rule changes [9]. Three cards were displayed in the form of a pyramid on the same screen (Figure $1 \mathrm{~B}$ ). On each white card, a diamond or a star shape was overlaid in red or blue. Participants were required to select which of the two cards at the bottom of the pyramid (selection cards) matched the card at the top of the pyramid (reference card) according to the given rule (shape or color). The color and shape of the two selection cards differed from those of the reference card. If the reference card was hemmed in black line, the correct response was to select the selection card that matched the reference card in shape. When no black hemming was shown, then the correct response was to select the selection card that matched the reference card in color. The reference and selection cards kept appearing on the screen until the participant responded, and the next set of cards appeared at random, $0.5 \mathrm{~s}$ after participants made their selection.

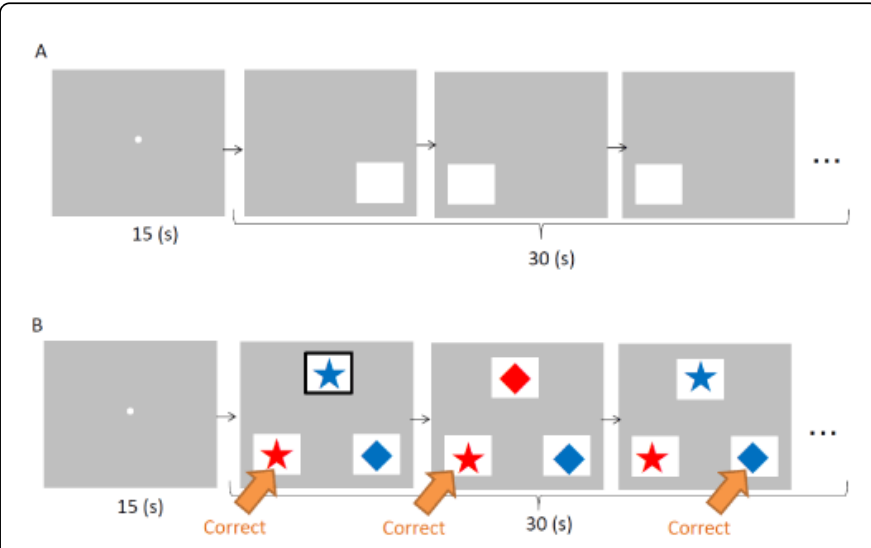

Figure 1: Illustration of the protocol for the baseline task (A) and Dimensional Change Card Sort (DCCS) task (B). In the baseline task, participants indicated the side of the screen on which the white square appeared. In the DCCS task, participants had to select the lower card with a symbol that matched the shape of the upper card symbol if the upper card was hemmed in black. If it was not hemmed in black, participants were required to select the lower card with a symbol of the same color as that on the upper card.
Participants were allowed a 15-s rest period before each successive trial. During the rest period, participants stared at a small white circle on the monitor screen, as in the baseline task. Task duration was $30 \mathrm{~s}$.

We performed the DCCS and baseline tasks twice, in alternation (i.e., baseline task i. DCCS task CC baseline task as DCCS task). In addition, before the actual task, participants underwent a practice trial (5-s rest period followed by a 20 -s trial). We provided feedback to participants during the practice trial and confirmed that participants completely understood the instructions. During the actual task, feedback was not provided, and participants' responses and reaction times were recorded automatically.

\section{Behavioral Data Analysis}

The number of correct answers and errors as well as the reaction times for correct responses and percentage of correct answers were assessed for each participant.

A significance level of $\mathrm{p}<0.05$ (two-sided) was adopted for all analyses.

\section{NIRS Recording and Analysis}

While participants performed the DCCS task, we recorded activity in the lateral $\mathrm{PFC}$ by noting changes in oxygenated hemoglobin $(\Delta \mathrm{HbO} 2)$ using a multi-channel NIRS system (OEG-16; Spectratech Inc., Tokyo, Japan). Near-infrared laser diodes emitted near-infrared light (approximately 770 and $840 \mathrm{~nm}$ ); re-emitted light was detected by avalanche photodiodes located $30 \mathrm{~mm}$ from the emitters with a temporal resolution of $655 \mathrm{~ms}$ at a measurement depth of approximately $3 \mathrm{~cm}$ below the scalp. In our system, six emitters and six detectors were placed at alternate points on a $2 \times 6$ grid (Figure. 2). This configuration enabled us to detect signals from 16 differential channels spread over the bilateral frontal region. The center of the probe matrix was placed on Fpz (International 10-10 system), and the bottom left and bottom right corners were located around F7 and F8,

respectively, in line with a previous report [23]. Detected NIRS signals were sent to a separate data collection computer. Presentation time of baseline symbols or DCCS cards was transmitted to the data collection computer from the task-control computer through a local area network with a UDP communication. A band-pass filter was set at 0.01-0.1 Hz during the task, using fast Fourier transformation to reject NIRS data distorted by minor movement artifacts. Data from each channel were converted into z-scores because raw data of highly variable amplitudes cannot be compared across participants and channels [15]. Z-scores were calculated using the mean and standard deviation of $\Delta \mathrm{HbO} 2$ during the last $6 \mathrm{~s}$ of the orienting period (when participants stared at a white dot at the center of the computer screen). The mean and standard deviation were adjusted to a $\mathrm{z}$-score of 0 and 1 for every channel. Trials with scores more than two standard deviations above or below the mean were excluded because of the possibility of motion artifacts. 
Citation: Yasumura A, Yamamoto H, Yasumura Y, Moriguchi Y, Hiraki K, et al. (2015) Cognitive Shifting in Children with Attention-Deficit Hyperactivity Disorder: A Near Infrared Spectroscopy Study. J Psychiatry 18: 196. doi:10.4172/2378-5756.1000196

Page 4 of 8



Figure 2: Near-infrared spectroscopy (NIRS) probes were attached to the scalp over the prefrontal cortex in the configuration shown here. The center of the probe matrix was placed on Fpz (International 10-10 system), and the bottom left and bottom right corners were located around F7 and F8, respectively.



Figure 3: Behavioral results of the Dimensional Change Card Sort task. The percentage of correct answers for each group during the task is plotted. Error bars indicate the standard error. ${ }^{*} \mathrm{p}<0.05$, ${ }^{* *} \mathrm{p}<0.01$

\section{Results}

\section{Behavioral results}

The percentage of correct answers $(t(57)=2.93, p<0.01$, Figure 3 ) and the number of errors $(\mathrm{t}(57)=2.80, \mathrm{p}<0.01$, Figure 4$)$ on the DCCS differed significantly between the ADHD and TDC groups; although,there were no differences in mean reaction times for correct responses $(t(57)=1.97, p>.05$, Figure 5).

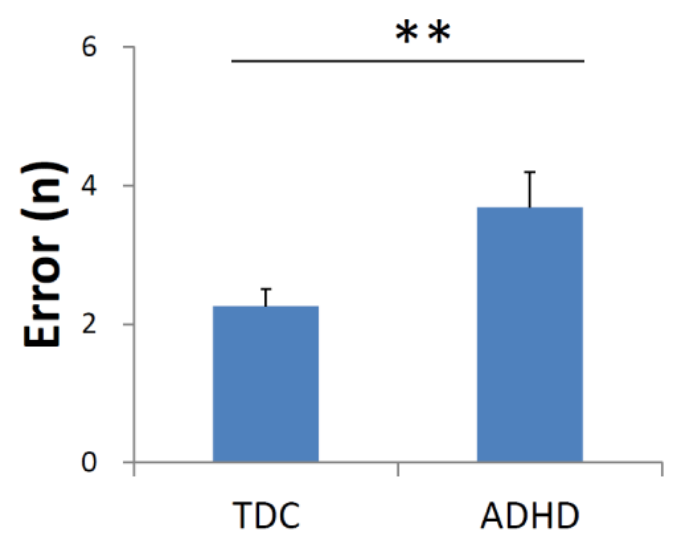

Figure 4: Behavioral results of the Dimensional Change Card Sort task. The number of errors for each group during the task is plotted. Error bars indicate the standard error. ${ }^{*} \mathrm{p}<0.05,{ }^{* *} \mathrm{p}<0.01$

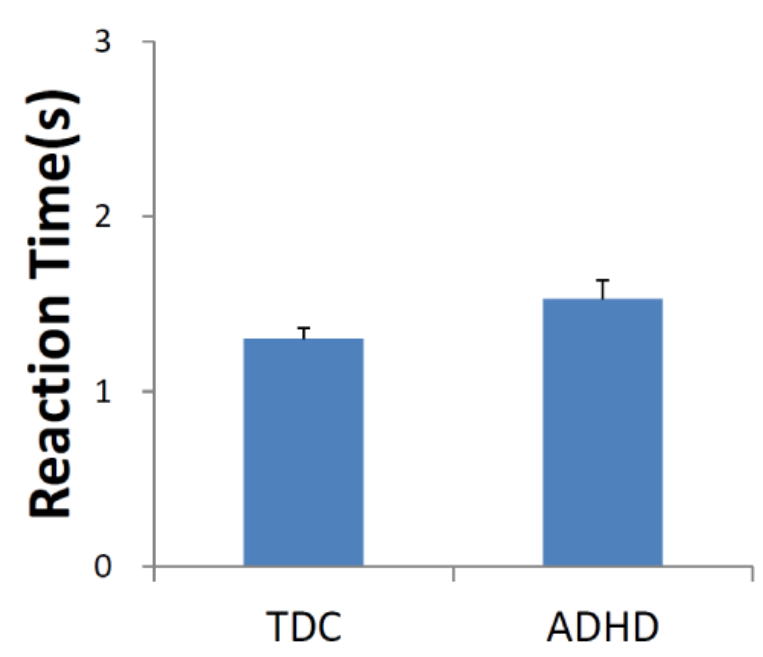

Figure 5: Behavioral results of the Dimensional Change Card Sort task. Reaction times for each group during the task are plotted. Error bars indicate the standard error. ${ }^{*} \mathrm{p}<0.05,{ }^{* *} \mathrm{p}<0.01$ 
Citation: Yasumura A, Yamamoto H, Yasumura Y, Moriguchi Y, Hiraki K, et al. (2015) Cognitive Shifting in Children with Attention-Deficit Hyperactivity Disorder: A Near Infrared Spectroscopy Study. J Psychiatry 18: 196. doi:10.4172/2378-5756.1000196

Page 5 of 8

\section{NIRS results}

During the baseline task, differences in the $\mathrm{HbO} 2$ signal were not observed between the ADHD and TDC groups. During the DCCS task, the $\mathrm{HbO} 2$ signal at channels 1,2 , and 13 were significantly lower in the ADHD group than in the TDC group $(\# 1: \mathrm{t}(57)=3.53, \mathrm{p}=$ $0.001, \# 2: \mathrm{t}(57)=2.46, \mathrm{p}=0.017, \# 13: \mathrm{t}(57)=2.22, \mathrm{p}=0.03$, Figure 6).

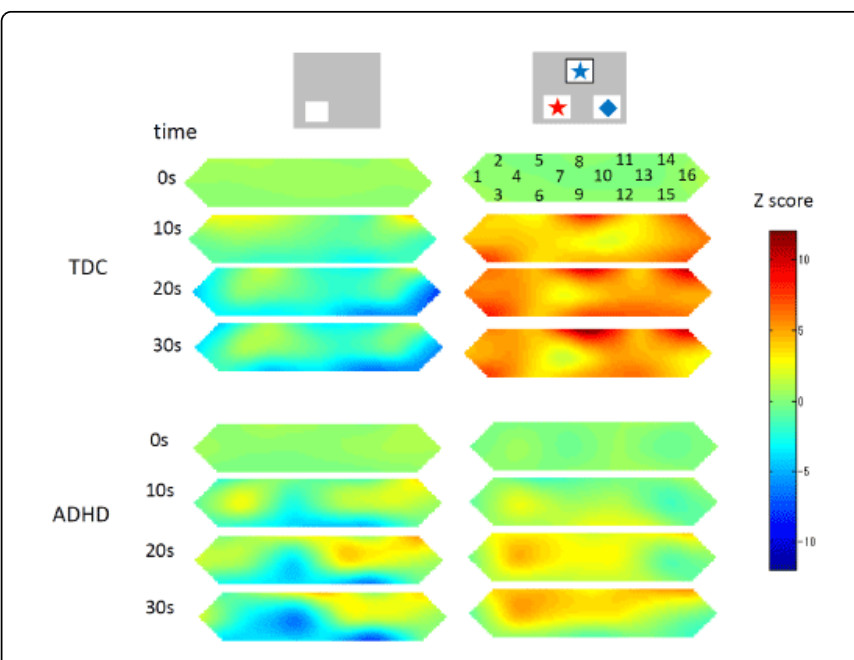

Figure 6: Time course of regional $\Delta \mathrm{HbO} 2$, as measured by nearinfrared spectroscopy, is shown for each group. The numbers on the first image of the second row indicate the locations of the channels. The left-side figure indicates prefrontal cortex (PFC) activity during the baseline task for TDC (upper) and ADHD (lower) groups. The right-side figure indicates PFC activity during the Dimensional Change Card Sort task for the TDC (upper) and ADHD (lower) groups.



Figure 7: Correlation of cortical activity measured by channel 1 (ch 1) with the Pervasive Developmental Disorders Autism Society Japan Rating Scale (PARS) infant score among all children.

\section{Correlations}

Negative correlations were observed between the $\mathrm{HbO} 2$ signal at channel $\# 1$ and both PARS scales (Infant: $\mathrm{r}=-0.30, \mathrm{p}<0.05$; Present: $\mathrm{r}=0-.29, \mathrm{p}<0.05$; Figures 7 and 8 , respectively). Moreover, negative correlations were observed between the $\mathrm{HbO} 2$ signal at channel \#1 and two of the SNAP subscales (inattention: $r=-0.39, \mathrm{p}<0.01$; hyperactivity/ impulsivity: $\mathrm{r}=0-.34, \mathrm{p}<0.01$; Figures 9 and 10, respectively). Positive correlations were observed between one of the SNAP subscales and number of errors (inattention: $\mathrm{r}=0.27, \mathrm{p}<0.05$; Figure 11 ).

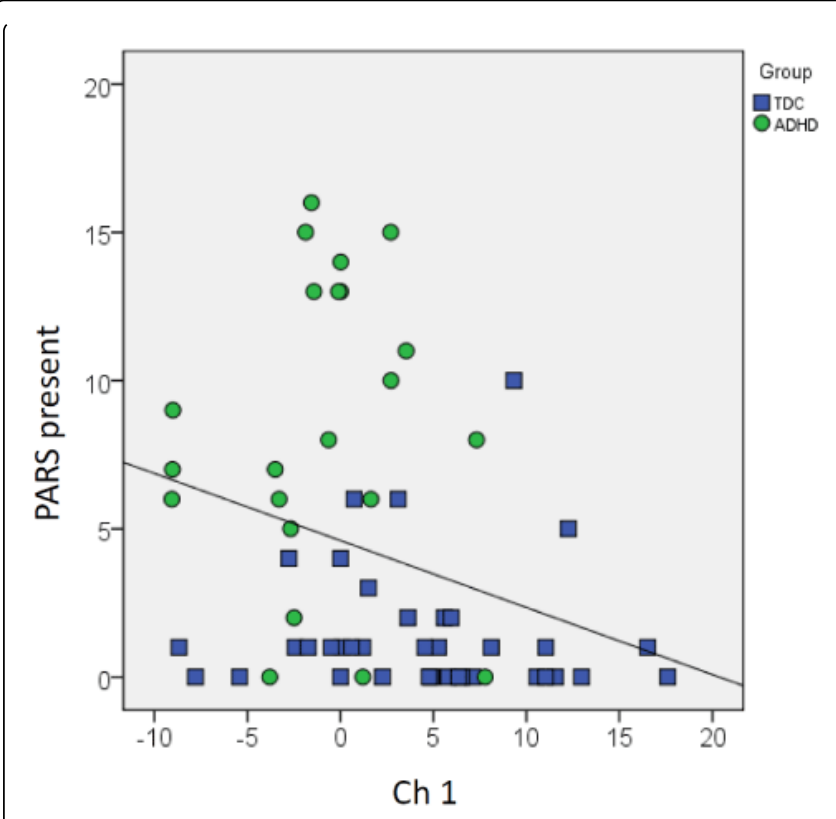

Figure 8: Correlation of cortical activity measured by channel 1 (ch 1) with the Pervasive Developmental Disorders Autism Society Japan Rating Scale (PARS) present score among all children.

\section{Discussion \\ Group differences in cognitive shifting}

During the DCCS task, children with ADHD made significantly more incorrect responses than did age- and IQ-matched normally developing children. Reaction times for all responses did not differ significantly between groups. These results indicate a deficit in cognitive shifting among children diagnosed with ADHD, consistent with previous studies [6]. The DCCS task requires participants to alter responses according to rule changes (color matching or shape matching). It is believed that higher numbers of incorrect responses reflect the inattention and hyperactivity characteristic of ADHD. In contrast, children with ADHD understood the rules of the task because their accuracy rate exceeded $75 \%$. In other words, the ADHD children understood the rules of the task but exhibited impairments in cognitive shifting. Compared with the TDC group, children with ADHD required more time to shift to the new rule, resulting in a greater number of incorrect responses. We believe that the DCCS task is more suitable to the evaluation of cognitive shifting than numerous other measures because its simplicity (i.e., only two classifications) minimizes the load on working memory. This contrasts with other tests of executive function, such as the WCST, which uses three classifications (color, shape, and number) [24]. In addition, the language impairment observed in some instances of ADHD should not affect the DCCS task because of its simple instructions and rules [25]. 
Citation: Yasumura A, Yamamoto H, Yasumura Y, Moriguchi Y, Hiraki K, et al. (2015) Cognitive Shifting in Children with Attention-Deficit Hyperactivity Disorder: A Near Infrared Spectroscopy Study. J Psychiatry 18: 196. doi:10.4172/2378-5756.1000196

Page 6 of 8

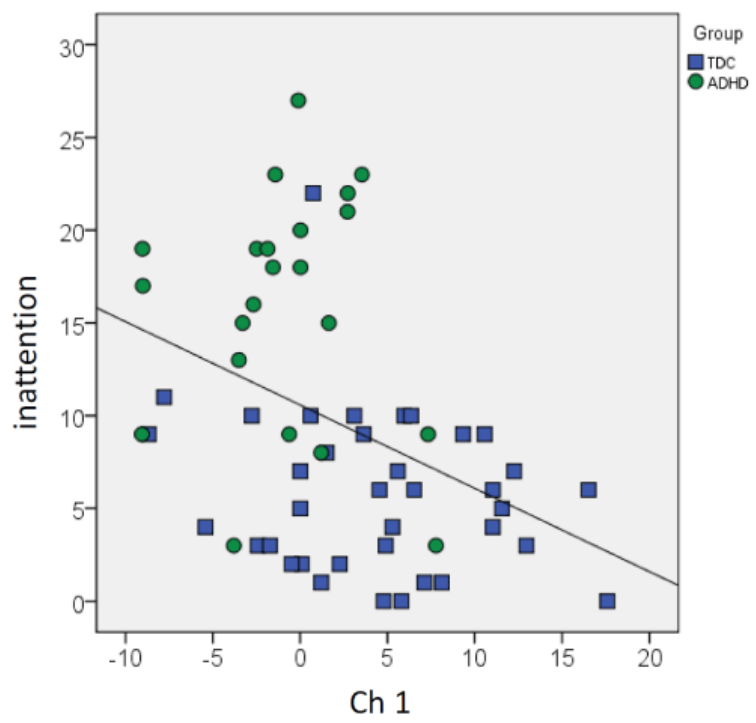

Figure 9: Correlation of cortical activity measured by channel 1 (ch 1) with the Swanson, Nolan, and Pelham scale (SNAP) inattention score among all children.

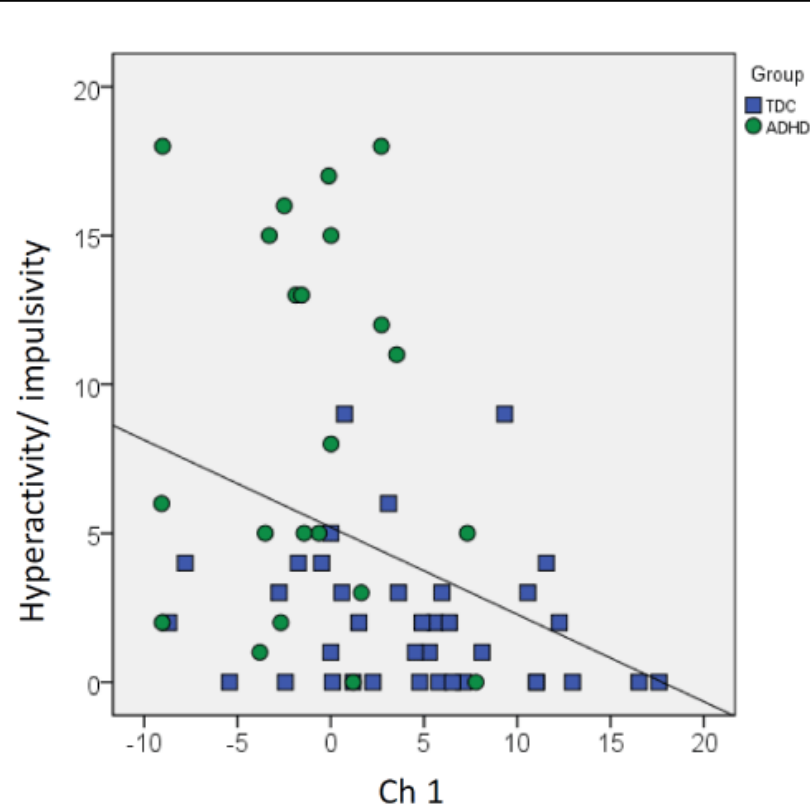

Figure 10: Correlation of cortical activity measured by channel 1 (ch 1) with the Swanson, Nolan, and Pelham scale (SNAP) hyperactivity/impulsivity score among all children.

\section{Neural findings}

Using NIRS, we found that cortical activity over channels 1,2 , and 13 was significantly lower in the ADHD group than in the TDC group during the DCCS task but not during the baseline task. The area surrounding regions \#1 and \#2 is the right inferior frontal gyrus (rIFG). This region is thought to play a significant role in inhibition [26-28]. Previous studies using an inhibition task (e.g., Stroop, Go/Nogo) have indicated that lack of inhibition is a core symptom of ADHD. In the present study, the larger number of errors made by children with ADHD may reflect problems with both inhibition and cognitive shifting. In addition, previous studies have suggested that children with ASD exhibit lower brain activation during cognitive shifting tasks $[14,29]$. Therefore, both ADHD and ASD seem to be associated with lower brain activation during this task. In order to clarify differences in the deficit, there is a need for further study of brain connectivity. For instance, the rIFG shows lower activation among three-year-old children who were unable to complete the DCCS task; this result was not found among five-year-old children [10]. Thus, because improvements in rIFG activity may not occur during natural development among individuals with ADHD, new methods for alleviating ADHD symptoms that target other brain areas are required.



Figure 11: Correlation of the number of errors with the Swanson, Nolan, and Pelham scale (SNAP) inattention score among children with attention-deficit/hyperactivity disorder (ADHD). 
Citation: Yasumura A, Yamamoto H, Yasumura Y, Moriguchi Y, Hiraki K, et al. (2015) Cognitive Shifting in Children with Attention-Deficit Hyperactivity Disorder: A Near Infrared Spectroscopy Study. J Psychiatry 18: 196. doi:10.4172/2378-5756.1000196

Page 7 of 8

\section{Correlations}

We observed correlations between ASD symptoms and brain activity among children with ADHD. These correlations are perhaps explained by the fact that developmental disorders make up a spectrum, whereby ASD and ADHD symptoms fall at different points

along the same continuum. These results may also be associated with the persistence of the DCCS task [14]. Furthermore, the correlations among ASD symptoms, ADHD, and brain activity may be associated with inhibition problems present among children with ADHD [28].

\section{Advantages and Limitations}

To measure activity of the prefrontal cortex during cognitive shifting, we measured $\triangle \mathrm{HbO} 2$ using NIRS, a neuroimaging technique employed in several previous studies to assess frontal lobe function among children and adults during the WCST. The NIRS technique has several advantages over functional magnetic resonance imaging (fMRI), most notably the lower cost and sensitivity to motion artifacts [8,30-32]. We note, however, that the NIRS detects hemodynamic changes only at the surface of the brain (approximately $3 \mathrm{~cm}$ beneath the skull). Thus, some potential differences in subcortical responses cannot be assessed using NIRS. Moreover, NIRS has a relatively low spatial resolution compared with fMRI. Despite these shortcomings, the NIRS is becoming a key imaging modality in developmental neuroscience studies involving newborns [33], preschool children [32], and school-aged children [15] because of its excellent safety and relative insensitivity to body movements. We believe that we can adequately measure neural activity patterns associated with executive function tasks using NIRS in children who have yet to develop full language capacity.

\section{Conclusions}

Deficits in cognitive shifting were observed among children with ADHD during the DCCS task. Children with ADHD exhibited more errors, as well as lower prefrontal cortex activity as compared to TDC. Overall, the DCCS task showed the presence of cognitive shifting deficits among children with ADHD.

\section{Acknowledgements}

This work was supported in part by the TMC Young Investigator Fellowship and an Intramural Research Grant (25-6; Clinical Research for Diagnostic and Therapeutic Innovations in Developmental Disorders) for Neurological and Psychiatric Disorders from the National Center of Neurology and Psychiatry (NCNP). This study was also supported by a Grant for Strategic Research Program for Brain Sciences (SRPBS: Project E) by the Ministry of Education, Culture, Sports, Science and Technology (Mizusawa $\mathrm{H}$ and Inagaki $\mathrm{M}$ ) of Japan. We thank the parents and children who participated in the study. We also thank the staff of the after-school care program of Sukage Nursery.

\section{Contributions}

All results were obtained through discussion among the authors. Every author reviewed the manuscript.

\section{Conflict of Interest}

The authors declare no conflicts of interest.

\section{References}

1. APA (2000) Diagnostic and Statistical Manual of Mental Disorders (4th ed. text rev.) Washington, D. C.

2. Barkley RA, Grodzinsky G, DuPaul GJ (1992) Frontal lobe functions in attention deficit disorder with and without hyperactivity: a review and research report. J Abnorm Child Psychol 20: 163-188.

3. Martel M, Nikolas M, Nigg JT (2007) Executive function in adolescents with ADHD. J Am Acad Child Adolesc Psychiatry 46: 1437-1444.

4. Pennington BF, Ozonoff S (1996) Executive functions and developmental psychopathology. J Child Psychol Psychiatry 37: 51-87.

5. Horowitz-Kraus T (2014) Pinpointing the deficit in executive functions in adolescents with dyslexia performing the Wisconsin card sorting test: an ERP study. J Learn Disabil 47: 208-223.

6. Kado Y, Sanada S, Yanagihara M, Ogino T, Ohno S, et al. (2012) Executive function in children with pervasive developmental disorder and attention-deficit/hyperactivity disorder assessed by the Keio version of the Wisconsin card sorting test. Brain Dev 34: 354-359.

7. Reeve WV, Schandler SL (2001) Frontal lobe functioning in adolescents with attention deficit hyperactivity disorder. Adolescence 36: 749-765.

8. Sumitani S, Tanaka T, Tayoshi S, Ota K, Kameoka N, et al. (2006) Activation of the prefrontal cortex during the Wisconsin Card Sorting Test as measured by multichannel near-infrared spectroscopy. Neuropsychobiology 53: 70-76.

9. Zelazo PD (2006) The Dimensional Change Card Sort (DCCS): a method of assessing executive function in children. Nat Protoc 1: 297-301.

10. Moriguchi Y, Hiraki K (2009) Neural origin of cognitive shifting in young children. ProcNatlAcad Sci U S A 106: 6017-6021.

11. Mulas F, Capilla A, Fernández S, Etchepareborda MC, Campo P, et al. (2006) Shifting-related brain magnetic activity in attention-deficit/ hyperactivity disorder. Biol Psychiatry 59: 373-379.

12. Bush G, Valera EM, Seidman LJ (2005) Functional neuroimaging of attention-deficit/hyperactivity disorder: a review and suggested future directions. Biol Psychiatry 57: 1273-1284.

13. Shaw P, Eckstrand K, Sharp W, Blumenthal J, Lerch JP, et al. (2007) Attention-deficit/hyperactivity disorder is characterized by a delay in cortical maturation. ProcNatlAcad Sci U S A 104: 19649-19654.

14. Yasumura A, Kokubo N, Yamamoto H, Yasumura Y, Moriguchi Y, et al. Neurobehavioral and hemodynamic evaluation of cognitive shifting in children with autism spectrum disorder. Behav Brain Sci 2: 463-470.

15. Matsuda G, Hiraki K (2006) Sustained decrease in oxygenated hemoglobin during video games in the dorsal prefrontal cortex: a NIRS study of children. Neuroimage 29: 706-711.

16. Shimada S, Hiraki K (2006) Infant's brain responses to live and televised action. Neuroimage 32: 930-939.

17. Wechsler D. (1991) Manual for Wechsler intelligence scale for childrenthird edition. The Psychological Corporation, San Antonio.

18. Raven J. (1976) Coloured progressive matrices: Sets A, Ab, B. OPP Ltd, Oxford.

19. Bussing R, Fernandez M, Harwood M, Wei Hou, Garvan CW, et al. (2008) Parent and teacher SNAP-IV ratings of attention deficit hyperactivity disorder symptoms: psychometric properties and normative ratings from a school district sample. Assessment 15: 317-328. 
Citation: Yasumura A, Yamamoto H, Yasumura Y, Moriguchi Y, Hiraki K, et al. (2015) Cognitive Shifting in Children with Attention-Deficit Hyperactivity Disorder: A Near Infrared Spectroscopy Study. J Psychiatry 18: 196. doi:10.4172/2378-5756.1000196

Page 8 of 8

20. Jensen P, Arnold L, Severe J, Vitiello B, Hoagwood K, et al. (2004) National Institute of Mental Health Multimodal Treatment Study of ADHD follow-up: 24-month outcomes of treatment strategies for attention-deficit/hyperactivity disorder. Pediatrics 113: 754-761.

21. Inoue Y, Ito K2, Kita Y3, Inagaki M3, Kaga M3, et al. (2014) Psychometric properties of Japanese version of the Swanson, Nolan, and Pelham, version-IV Scale-Teacher Form: a study of school children in community samples. Brain Dev 36: 700-706.

22. Ogata H, Ihara H, Murakami N, Gito M, Kido Y, et al. (2014) Autism spectrum disorders and hyperactive/impulsive behaviors in Japanese patients with Prader-Willi syndrome: a comparison between maternal uniparentaldisomy and deletion cases. Am J Med Genet A 164A: 2180-2186.

23. Kita Y, Gunji A, Inoue Y, Goto T, Sakihara K, et al. (2011) Self-face recognition in children with autism spectrum disorders: a near-infrared spectroscopy study. Brain Dev 33: 494-503.

24. Watson TD, Azizian A, Squires NK (2006) Event-related potential correlates of extradimensional and intradimensional set-shifts in a modified Wisconsin Card Sorting Test. Brain Res 1092: 138-151.

25. Kjelgaard MM, Tager-Flusberg H (2001) An Investigation of Language Impairment in Autism: Implications for Genetic Subgroups. Lang Cogn Process 16: 287-308.

26. Menon V, Adleman NE, White CD, Glover GH, Reiss AL (2001) Errorrelated brain activation during a $\mathrm{Go} / \mathrm{NoGo}$ response inhibition task. Hum Brain Mapp 12: 131-143.
27. Tamm L, Menon V, Ringel J, Reiss AL (2004) Event-related FMRI evidence of frontotemporal involvement in aberrant response inhibition and task switching in attention-deficit/hyperactivity disorder. J Am Acad Child Adolesc Psychiatry 43: 1430-1440.

28. Yasumura A, Kokubo N2, Yamamoto H2, Yasumura Y3, Nakagawa E4, et al. (2014) Neurobehavioral and hemodynamic evaluation of Stroop and reverse Stroop interference in children with attention-deficit/ hyperactivity disorder. Brain Dev 36: 97-106.

29. Shafritz KM, Dichter GS, Baranek GT, Belger A (2008) The neural circuitry mediating shifts in behavioral response and cognitive set in autism. Biol Psychiatry 63: 974-980.

30. Sanefuji M, Takada Y, Kimura N, Torisu H, Kira R, et al. (2011) Strategy in short-term memory for pictures in childhood: a near-infrared spectroscopy study. Neuroimage 54: 2394-2400.

31. Schroeter ML, Zysset S, Wahl M, von Cramon DY (2004) Prefrontal activation due to Stroop interference increases during development--an event-related fNIRS study. Neuroimage 23: 1317-1325.

32. Tsujimoto S1, Yamamoto T, Kawaguchi H, Koizumi H, Sawaguchi T (2004) Prefrontal cortical activation associated with working memory in adults and preschool children: an event-related optical topography study. Cereb Cortex 14: 703-712.

33. Otsuka Y, Nakato E, Kanazawa S, Yamaguchi MK, Watanabe S, et al. (2007) Neural activation to upright and inverted faces in infants measured by near infrared spectroscopy. Neuroimage 34: 399-406. 